\title{
Weeds in Greenhouses and Tobacco Fields Are Differentially Infected by Tomato spotted wilt virus and Infested by Its Vector Species
}

\author{
E. K. Chatzivassiliou, I. Boubourakas, Plant Pathology Laboratory, Faculty of Agriculture, Aristotle University, \\ Thessaloniki, Greece; E. Drossos, Laboratory of Systematic Botany and Phytogeography, School of Biology, Aris- \\ totle University, Thessaloniki; I. Eleftherohorinos, Department of Agronomy, Faculty of Agriculture, Aristotle \\ University, Thessaloniki; G. Jenser, Plant Protection Institute, Hungarian Academy of Sciences, Budapest, Hun- \\ gary; D. Peters, Department of Virology, Agricultural University of Wageningen, The Netherlands; and N. I. Katis, \\ Plant Pathology Laboratory, Faculty of Agriculture, Aristotle University, Thessaloniki
}

\begin{abstract}
Chatzivassiliou, E. K., Boubourakas, I., Drossos, E., Eleftherohorinos, I., Jenser, G., Peters, D., and Katis, N. I. 2001. Weeds in greenhouses and tobacco fields are differentially infected by Tomato spotted wilt virus and infested by its vector species. Plant Dis. 85:40-46.

A survey was conducted in the Macedonia region of Greece to determine the reservoir hosts of Tomato spotted wilt virus (TSWV) in three tobacco fields and in a greenhouse complex in which lettuce and the ornamentals chrysanthemum, gerbera, aster, and anemone were grown. Assays for TSWV infection were made by enzyme-linked immunosorbent assay on 6,172 plant samples, 3,909 from tobacco fields and 2,263 from the greenhouse complex, comprising plants of 208 species in 137 genera of 42 families. Plants of 86 species out of 63 genera of 27 families were infected of which 39 species are newly reported hosts of TSWV. An infection index was developed to evaluate the relative potential of each weed species as a virus source in both systems. Seventeen species in the tobacco fields and nine in the greenhouses had an infection index higher than one. Most species with infected plants were found in the Compositae family. Plants of some species occurring both in tobacco fields and in greenhouses were infected at only one of these sites. Frankliniella occidentalis was the common thrips species on weeds and crops in the greenhouses, while Thrips tabaci was the only vector on tobacco plants and weeds in the tobacco fields. This observation strongly suggests that the occurrence of species with infected plants and their number have to be attributed to the vector species prevailing in the greenhouse complex or tobacco fields, supporting the conclusion that TSWV is spread in two different epidemiological processes in Greece.
\end{abstract}

Additional keywords: ELISA, hosts, Thripidae, transmission efficiency

Tomato spotted wilt virus (TSWV) (family Bunyaviridae, genus Tospovirus) is one of the most devastating plant viruses that cause diseases in many economically important crops and often tremendous crop losses worldwide. In Greece, TSWV was initially described in tobacco crops in the regions of Central and East Macedonia by Tsakiridis and Gooding in 1972 (35). Since 1990, after the worldwide expansion of Frankliniella occidentalis Pergande, this virus has caused serious problems in the production of greenhouse and field-grown vegetable and ornamental crops $(8,9)$. Before the introduction of $F$. occidentalis, the only vector of TSWV in Greece was considered to be Thrips tabaci Lindeman. F. occidentalis, first reported in Crete (27) and soon thereafter on the mainland, was

Corresponding author: N. I. Katis

E-mail: katis@agro.auth.gr

Accepted for publication 6 September 2000.

Publication no. D-2000-1025-03R

(C) 2001 The American Phytopathological Society associated with outbreaks of TSWV infections in vegetable and ornamental crops in several parts of Greece.

The ability of tospovirus vectors (Thysanoptera: Thripidae) to infest plants of many species is one of the main reasons that TSWV has a wide natural host range. More than 1,050 species of at least 92 botanical families, both monocots and dicots, have been reported susceptible to tospoviruses of which more than 900 species can host TSWV (23). Its wide host range, including many species grown as vegetables and ornamentals, increases the difficulty of controlling this virus. Many, if not all, of them are also hosts for the thrips species vectoring tospoviruses.

Since tospoviruses are not seed transmitted (26), infected weeds are probably the main reservoirs from which the virus is spread to susceptible crops. The potential of this reservoir depends on the number of infected weed plants as well as their infestation by thrips.

This paper presents results of a survey to collect information on the occurrence of infected weeds and their infestation by vectors in two different crop systems. One system consisted of horticultural crops grown in a greenhouse complex in which $F$. occidentalis prevails as vector and the other of tobacco crops where $T$. tabaci transmits exclusively TSWV. Implications of the role of the identified hosts on the spread of TSWV are discussed.

\section{MATERIALS AND METHODS}

Surveys for virus incidence. Weeds were sampled from September 1995 to September 1998 in three tobacco fields of Thessaloniki, Kilkis, and Drama, the major tobacco growing areas of the northern Greek region of Macedonia. Weed samples were also taken from a greenhouse complex in Chalkidiki, one of the main vegetable and ornamental producing regions of northern Greece, where lettuce, chrysanthemum, gerbera, aster, and anemone were grown. The incidence of TSWV in these regions was high during the 1990s (E. K. Chatzivassiliou and N. I. Katis, unpublished data).

In total 6,172 plants, 3,909 from tobacco fields and 2,263 from the greenhouse complex, belonging to 208 species of 137 genera from 42 families were randomly collected. Plants in the borders of the tobacco fields and between the greenhouse units were also sampled. The material sampled consisted mainly of the youngest fully expanded leaf or young leaves and flowers, when present.

The species from which leaf material was sampled were identified in the Laboratory of Systematic Botany and Phytogeography, and the Weed Science section of the Laboratory of Agronomy.

TSWV detection. Samples were analyzed for TSWV infection by double antibody sandwich enzyme-linked immunosorbent assay (DAS-ELISA). Tests with polyclonal antiserum raised against the nucleocapsid $(\mathrm{N})$ protein of a local TSWV isolate were carried out using flat-bottom microplates (EIA/RIA Costar Corporation, Cambridge, MA) (38). Antibodies and conjugated antibodies were used in a concentration of $1 \mu \mathrm{g}$ per ml. Extracts were prepared by grinding leaf tissue at a ratio of 1:200 (wt/vol) in freshly prepared phosphate-buffered saline-Tween with $2 \%$ (wt/vol) polyvinylpyrollidone (molecular 
weight: 6000). Substrate ( $p$-nitrophenyl phosphate, Sigma, Steinheim, Germany) was used at a concentration of $1 \mathrm{mg}$ per $\mathrm{ml}$. The absorbance values, read on a microplate ELISA-reader (MPR-AA2, Eurosystem, Tessenderlo, Belgium) at $\mathrm{OD}_{405}$ $\mathrm{nm}$, were corrected for blank values consisting of only buffer in the sample incubation step. Samples with values higher than twice the average ELISA values of samples from healthy plants were considered positive. Healthy tobacco plants grown in the laboratory and plants of the tested species, collected on the farm of the Agricultural Faculty of Aristotle University of Thessaloniki, where no TSWV infections were found, were used as controls.

Samples positive for TSWV were mechanically inoculated onto Nicotiana rustica and Petunia hybrida plants to confirm the authencity of the virus. Inoculations were made using a $0.01 \mathrm{M}$ phosphate buffer, $\mathrm{pH} 7.0$, containing $0.1 \%$ sodium sulfite, and $1 \%$ activated charcoal to indicator plants. After inoculation, plants were transferred to a glasshouse for symptom development. Infection by TSWV was confirmed by development of local lesions on $P$. hybrida in 3 to 4 days, or a typical mosaic on $N$. rustica within 2 weeks.

Estimation of the importance of weeds as a possible virus source. The contribution of the infected plants of each species to the infection pressure of the weed flora in the tobacco or greenhouse crops was defined as the "relative infection potential index." This index was expressed as the fraction of infected plants of a species over the infected plants of all species multiplied with the fraction of all sampled plants of the species over all sampled plants of all species and described by the formula:

\section{$I=[P S / P A S] *[I P / I P A] / \sum_{i=1}^{n}[(P S / P A S) *$ \\ $(I P / I P A)] i * 100$}

in which $P S=$ number of plants of a species sampled in a crop system, $P A S=$ number of plants of all host species sampled in a crop system, $I P=$ number of infected plants of a species in a crop system, and $I P A=$ number of infected plants of all host species in a crop system.

Thrips collection and identification. To identify the thrips species infesting the weeds and crop plants in the greenhouse complex and tobacco fields, whole plants were collected and sealed in plastic bags during the survey. Thrips were tapped from the plants on a white tray, collected in vials containing AGA (10 parts of $60 \%$ ethanol, one part each of glycerine and acetic acid) (22), and identified.

Infectivity of thrips infesting the weeds. The role of weeds as TSWV reservoirs was further evaluated by the percentage of infective vector thrips infesting these plants in the tobacco fields and greenhouse complex. In the first year, the thrips from all plants of a species were collected from a TSWV-infected tobacco field or from a blooming chrysanthemum greenhouse crop and pooled. In the second year, plants were individually assayed for thrips infestation and virus infection. The adults were collected by tapping the plants from the tobacco fields or greenhouse on a white tray. Their transmission ability was individually tested on leaf disks of Nicotiana tabacum variety Basmas BZ/7 or $P$. hybrida plants, in two successive inoculation periods of $48 \mathrm{~h}$ at $25^{\circ} \mathrm{C}$, while kept in a light-dark regime of 16 to $8 \mathrm{~h}$ (38). The petunia leaf disks were visually checked for local lesion development, whereas the tobacco disks were individually analyzed by ELISA after 5 days of incubation while floating on water. The number of infected disks was used to calculate infection rates.

The weed plants, on which the thrips were collected, were tested for TSWV infection by ELISA, and samples of 10 thrips from each plant species were used for identification.

\section{RESULTS}

Weed plants as hosts of TSWV and their importance in tobacco and greenhouse crops. Assays showed that many species growing in and near tobacco fields and greenhouses harbor TSWV. Plants of 86 species in 63 genera belonging to 27 families were positive in ELISA. Infection was confirmed in plants of 52 species by mechanical inoculation to petunia and $N$. rustica. Most infected plants belonged to species in the Compositae family followed by the Solanaceae, Labiatae, and Plantaginaceae families (Tables 1 and 2).

Infected plants of species of Aster, Carduus, Convolvulus, Galium, Lamium, Portulaca, Sonchus, Trifolium, and Vicia were found in the greenhouses, but not in the tobacco crops (Tables 1 and 2). Plants of Amaranthus, Anthemis, Capsella, Chaerophyllum, Chamomilla, Conium, Cynodon, Fumaria, Papaver, Polygonum, and Rumex appeared to be infected in tobacco crops, but not in the greenhouses (Tables 1 and 2). Plants of Ballota, Cirsium, Conyza, Datura, Lactuca, Malva, Picris, Potentilla, Senecio, Silybum, Sinapis, Solanum, Sonchus, Stellaria, Tribulus, Veronica, and Xanthium were found to be infected in the greenhouses as well as in the tobacco fields.

Species where more than $50 \%$ of the plants were infected in the greenhouses were species of Potentilla, Xanthium, and Aster (Table 1). More than 50\% of the plants belonging to Anthemis, Chamimilla, Silybum, Sonchus, Veronica, Cirsium, Veronica, Mentha, Chenopodium, Picris, Sanguisorba, Cirsium, and Cynoglossum were infected in the tobacco crops (Table 2). The number of plants of these species, however, was often low.

Genera most frequently occurring in greenhouses were Senecio, Sonchus, Stel- laria, Convolvulus, Galium, Solanum, Aster, Sinapis, and Solanum (Table 1), and in the tobacco fields Solanum, Plantago, Amaranthus, Capsella, Ballota, Artemisia, Datura, and Papaver (Table 2).

Virus could not be detected by ELISA or bioassays in plants of 122 species from 36 families, either occurring in the greenhouses, tobacco fields, or both. These species were Amaranthus albus (20 plants tested), A. blitoides (31), Daucus carota (5), Eryngium campestre (5), Orlaya sp. (3), Pimpinella sp. (8), Torilis japonica (2), Anchusa officinalis (8), A. undulata (2), Asperugo procumbens (5), Buglossoides arvensis (3), Symphytum sp. (1), S. officinale (9), S. tuberosum (9), Legousia speculum (3), Humulus lupulus (2), Sambucus elubus (3), Herniaria hirsuta (3), Lychnis coronaria (16), Silene densiflora (6), S. vulgaris (1), Chenopodium amaranticolor (27), Calendula officinalis (6), Carduus pycnocephalus (1), Crepis setosa (2), Hedypnois cretica (1), Helianthus tuberosus (3), Hypochoeris radicata (2), Matricaria sp. (10), M. perforata (4), Onopordun illyricon (15), Picris sprengerana (3), Taraxacum officinale (14), Tragopogon porifolius (4), Convolvulus althaeoides (1), Cornus sanguinea (6), Alyssum chalcidicum (5), Cardaria draba (96), Diplotaxis tenuifolia (1), Lepidium sp. (7), Nasturtium officinale (6), Sinapis sp. (1), Sisymbrium sp. (4), Cyperus rotundus (56), Cephalaria transylvanica (15), Equisetum arvense (3), Chrozophora tinctoria (1), Euphorbia sp. (7), E. chamaesyce (2), E. cyparissias (16), E. exigua (7), E. helioscopia (14), Galega officinalis (4), Melilotus alba (11), Vicia tenuifolia (14), Geranium sp. (5), G. rotundifolium (5), Aegilops triuncialis (5), Avena sativa (2), A. sterilis (12), Cynodon dactylon (17), Dactylis glomerata (4), Lolium sp. (4), Poa bulbosa (2), P. trivialis (3), Setaria verticillata (7), Setaria viridis (18), Sorghum halepense (17), Hypericum crispum (6), $H$. perforatum (10), Lamium sp. (8), Marrubium sp. (2), Melissa officinalis (12), Mentha sp. (24), M. arvensis (1), Nepeta nuda (1), Prunella vulgaris (7), Salvia sp. (2), S. pratensis (5), S. verbenaca (23), Sideritis montana (12), Stachys germanica (2), Thymus sipthorpii (1), Asphodelus microcarpus (1), Ruscus aculeatus (3), Abutilon theophrasti (4), Malva sp. (8), M. pusilla (10), M. sylvestris (3), Acacia sp. (1), Jasminum fruticans (1), Epilobium hirsutum (4), Fumaria sp. (4), Bilderdykia convolvulus (25), Rumex crispus (22), Consolida regalis (17), Helleborus cyclophyllus (9), Ranunculus sardous (2), Anagalis arvenis L. (3), Agrimonia sp. (2), A. agrimonoides (1), A. eupatoria (26), Crataegus monogyna (19), Geum urbanum (2), Potentilla sp. (1), P. micrantha (7), Prunus insititia (6), Pyrus pyraster (1), Rosa canina (7), Rubus sp. (12), R. canescens (4), R. ulmifolius (19), Galium verum (2), Kickxia elatine (4), Verbascum blat- 
taria (6), V. flomoides (3), V. sinuatum (4), Veronica hederifolia (7), Hyoscyamus albus (1), Urtica sp. (11), U. dioica (21), and Viola sp. (2).

In many cases, the infected weeds did not show virus symptoms. Symptoms characteristic for TSWV, like chlorotic or necrotic ring spots, mosaic or mottling, leaf malformation, and stunting were found on some infected plants of Amaranthus retroflexus, Anchusa arvensis, Aristolochia clematitis, Aster sp., Chenopodium album, Cichorium intybus, Cirsium arvense, Clematis flamula, $C$. vitalba, Conyza canadensis, Datura stramonium, Heliotropium europaeum, Lactuca serriola, Malva neglecta, Plantago major, P. lanceolata, Senecio vulgaris, Scabiosa tenuis, Solanum nigrum, Sonchus sp., S. asper, S. arvense, S. oleraceus, and Xanthium strumarium.

All plants of the species Orlaya sp., Anchusa arvensis, A. undulata, Symphytum tuberosum, Onopordun illyricon, Cornus sanguinea, Melissa officinalis, Nepeta nuda, Agrimonia eupatoria, Geum urbanum, Rubus canascens, R. ulmifolius, Rubus sp., Galium verum, and Verbascum blattaria gave high optical density (OD) values even though no virus could be detected. Similar results were obtained when plants of the same species were collected from areas where no TSWV could be detected. These plants were, therefore, considered to be healthy, and the positive
ELISA reactions were explained by a strong nonspecific reaction. Virus could be recovered in one case by mechanical inoculation from an Anchusa arvensis plant showing ring spots. This plant gave high OD values like apparently healthy plants from which no virus could be recovered.

Weed infestation by thrips. In greenhouses, low numbers of thrips were collected on plants of all weed species compared to chrysanthemum flowers. Adults and larvae of $F$. occidentalis were found on lettuce, chrysanthemum, gerbera, aster, anemone, and on the weeds Senecio vulgaris, Sonchus sp. (Compositae), Datura stramonium, Solanum elaeagnifolium, and $S$. nigrum (Solanaceae). In the greenhouses, T. tabaci was found only on Conyza canadensis, while T. tabaci and $F$. occidentalis adults were found on Xanthium spinosum. Other thrips species, such as Thrips sp. and Haplothrips sp., were found on healthy Silybum marianum plants in the greenhouses. These species are not recorded as vectors of tospoviruses. No thrips were found on Cyperus rotundus (5 plants), Conyza canadensis (3 plants), and Lactuca serriolla (3 plants).

T. tabaci constituted the predominant thrips species in the tobacco fields and was the only vector on weeds and tobacco plants. This thrips was found on the susceptible TSWV species Amaranthus retroflexus (Amaranthaceae), Cirsium arvense,
Conyza cannadensis, Echinops ritro, Sonchus sp., Xanthium spinosum, X. strumarium (Asteraceae), Datura stramonium, Solanum nigrum, (Solanaceae), Tribullus terrestris (Zygophylaceae), and the nonsusceptible species Kichxia elatine (Scrophulariaceae) and Sorghum halepense (Graminae or Poaceae). Larvae of unidentified thrips species and occasionally adults of Aeolothrips spp., Limothrips cerealium, L. denticornis, Taeniothrips spp., and Thrips spp., which are not known to vector TSWV, were also collected from these plant species.

In tobacco fields, most thrips were found on the flowers of Amaranthus retroflexus plants, but some lived on both sides of the leaves. Thrips occurred mainly in the existing flowers of Xanthium strumarium and $X$. spinosum plants, and when high populations occurred, they were also found around the base of the seeds and a few on the underside of the top leaves. On Solanum nigrum plants, thrips were found on the upper side of the leaves, which were damaged by feeding scars. Males were the predominant sex on Echinops ritro plants. No thrips were found on the single Chondrila juncea and Cirsium arvense plant encountered or on 10 Conyza canadense plants after the blooming season. They also were not found on two plants of Galium lusidum; three plants each of Conium maculatum, Scabiosa tenuis and Artemisia

Table 1. The incidence of Tomato spotted wilt virus in infected annual (An), biennial (Bi), and perennial (Pe) weed species and their relative infection potential index in greenhouse crops

\begin{tabular}{|c|c|c|c|c|c|}
\hline Family & Species $^{\mathrm{a}}$ & Life span & $\begin{array}{l}\text { Number of plants in- } \\
\text { fected/tested }\end{array}$ & $\begin{array}{l}\% \text { of plants in sam- } \\
\text { pled population }\end{array}$ & $\begin{array}{l}\text { Relative infection po- } \\
\text { tential index }\end{array}$ \\
\hline Caryophyllaceae & Stellaria media $^{\mathrm{b}}$ & $\mathrm{Bi}(\mathrm{Pe})$ & $14 / 143$ & 6.3 & 8.83 \\
\hline \multirow[t]{14}{*}{ Compositae } & Aster sp. ${ }^{\mathrm{b}}$ & & $52 / 79$ & 3.5 & 18.13 \\
\hline & Carduus nutans ${ }^{b}$ & $\mathrm{Bi}$ & $1 / 8$ & 0.4 & 0.04 \\
\hline & Cirsium arvense $^{\mathrm{b}}$ & $\mathrm{Pe}$ & $10 / 70$ & 3.1 & 3.09 \\
\hline & Conyza canadensis ${ }^{\mathrm{b}}$ & An & $18 / 54$ & 2.4 & 4.29 \\
\hline & Lactuca serriola ${ }^{\mathrm{b}}$ & $\mathrm{Bi}(\mathrm{An})$ & $2 / 17$ & 0.8 & 0.15 \\
\hline & Picris sp. & & $2 / 9$ & 0.4 & 0.08 \\
\hline & Senecio vulgaris ${ }^{\mathrm{b}}$ & An & $31 / 251$ & 11.1 & 34.33 \\
\hline & Silybum marianum $^{\mathrm{b}}$ & $\mathrm{Bi}$ & $4 / 17$ & 0.5 & 0.3 \\
\hline & Sonchus arvensis ${ }^{\mathrm{b}}$ & $\mathrm{Pe}$ & $4 / 30$ & 1.0 & 0.53 \\
\hline & Sonchus oleraceus ${ }^{\mathrm{b}}$ & $\mathrm{An}(\mathrm{Bi})$ & $18 / 22$ & 1.3 & 1.75 \\
\hline & Sonchus sp. ${ }^{\mathrm{b}}$ & & $18 / 155$ & 6.9 & 12.31 \\
\hline & Tagetes sp. & & $4 / 9$ & 0.4 & 0.16 \\
\hline & Xanthium spinosum ${ }^{\mathrm{b}}$ & An & $6 / 7$ & 0.3 & 0.19 \\
\hline & Xanthium strumarium ${ }^{\mathrm{b}}$ & An & $9 / 11$ & 0.5 & 0.44 \\
\hline \multirow[t]{2}{*}{ Convolvulaceae } & Convolvulus arvensis ${ }^{\mathrm{b}}$ & $\mathrm{Pe}$ & $10 / 120$ & 5.3 & 5.29 \\
\hline & Ipomea purpurea $^{\mathrm{b}}$ & An & $1 / 16$ & 0.7 & 0.07 \\
\hline Cruciferae & Sinapis arvensis & An & $3 / 76$ & 3.4 & 1.01 \\
\hline \multirow[t]{2}{*}{ Fabaceae } & Trifolium sp. & & $2 / 27$ & 1.3 & 0.24 \\
\hline & Vicia sp. & & $1 / 20$ & 0.9 & 0.09 \\
\hline \multirow[t]{2}{*}{ Labiatae } & Ballota nigra & $\mathrm{Pe}$ & $5 / 18$ & 0.8 & 0.4 \\
\hline & Lamium amplexicaule $^{\mathrm{b}}$ & An & $1 / 70$ & 3.1 & 0.31 \\
\hline Malvaceae & Malva neglecta & An & $2 / 27$ & 1.2 & 0.24 \\
\hline Portulacaceae & Portulaca oleraceae ${ }^{\mathrm{b}}$ & An & $3 / 52$ & 2.3 & 0.69 \\
\hline Rosaceae & Potentilla reptans & $\mathrm{Pe}$ & $2 / 2$ & 0.1 & 0.02 \\
\hline Rubiaceae & Galium aparine $^{\mathrm{b}}$ & $\mathrm{An}$ & $1 / 113$ & 5.0 & 0.5 \\
\hline Scrophulariaceae & Veronica officinalis ${ }^{b}$ & $\mathrm{Pe}$ & $2 / 9$ & 0.4 & 0.08 \\
\hline \multirow[t]{3}{*}{ Solanaceae } & Datura stramonium ${ }^{\mathrm{b}}$ & An & $4 / 13$ & 0.6 & 0.23 \\
\hline & Solanum elaeagnifoliumb & $\mathrm{Pe}$ & $1 / 73$ & 3.2 & 0.32 \\
\hline & Solanum nigrum ${ }^{\mathrm{b}}$ & An & $13 / 102$ & 4.5 & 5.85 \\
\hline Zygophylaceae & Tribulus terrestris & An & $2 / 8$ & 0.4 & 0.07 \\
\hline
\end{tabular}

\footnotetext{
a Newly identified host species are given in bold.

${ }^{\mathrm{b}}$ Infection confirmed by mechanical inoculation to petunia and $N$. rustica.
} 
Table 2. Incidence of Tomato spotted wilt virus infected annual (An), biennial (Bi), and perennial (Pe) weeds and their relative infection potential index in tobacco crops

\begin{tabular}{|c|c|c|c|c|c|}
\hline Family & Species $^{\mathrm{a}}$ & Life span & $\begin{array}{l}\text { Number of plants } \\
\text { infected/tested }\end{array}$ & $\begin{array}{c}\% \text { of plants in } \\
\text { sampled population }\end{array}$ & $\begin{array}{l}\text { Relative infection poten- } \\
\text { tial index }\end{array}$ \\
\hline Amaranthaceae & Amaranthus retroflexus ${ }^{\mathrm{b}}$ & An & $3 / 144$ & 3.7 & 0.65 \\
\hline \multirow[t]{4}{*}{ Apiaceae } & Chaerophyllum sp. & & $22 / 45$ & 1.2 & 1.49 \\
\hline & Chaerophyllum temulentum & An (Bi) & $6 / 10$ & 0.3 & 0.09 \\
\hline & Conium maculatum & $\mathrm{Pe}$ & $1 / 6$ & 0.2 & 0.01 \\
\hline & Torilis sp. & An & $1 / 7$ & 0.2 & 0.01 \\
\hline Araceae & Arum maculatum $^{\mathrm{b}}$ & $\mathrm{Pe}$ & $1 / 67$ & 1.7 & 0.10 \\
\hline Aristolochiaceae & Aristolochia clematitis ${ }^{\mathrm{b}}$ & $\mathrm{Pe}$ & $11 / 84$ & 2.2 & 1.39 \\
\hline \multirow[t]{3}{*}{ Boraginaceae } & Anchusa arvensis & An & $1 / 11$ & 0.5 & 0.02 \\
\hline & Cynoglossum sp. & & $1 / 2$ & 0.1 & 0.00 \\
\hline & Heliotropium europaeum ${ }^{\text {b }}$ & An & $3 / 10$ & 0.3 & 0.05 \\
\hline \multirow[t]{4}{*}{ Caryophyllaceae } & Saponaria officinalis & $\mathrm{Pe}$ & $1 / 6$ & 0.2 & 0.01 \\
\hline & Silene alba & $\mathrm{Bi}(\mathrm{Pe})$ & $1 / 18$ & 0.5 & 0.03 \\
\hline & Silene sp. & & $1 / 10$ & 0.3 & 0.02 \\
\hline & Stellaria media ${ }^{\mathrm{b}}$ & $\mathrm{Bi}(\mathrm{Pe})$ & $1 / 5$ & 0.1 & 0.01 \\
\hline \multirow[t]{2}{*}{ Chenopodiaceae } & Chenopodium album ${ }^{\mathrm{b}}$ & An & $3 / 61$ & 1.4 & 0.28 \\
\hline & Chenopodium glaucum ${ }^{\mathrm{b}}$ & An & $9 / 13$ & 0.3 & 0.18 \\
\hline \multirow[t]{24}{*}{ Compositae } & Anthemis arvensis ${ }^{\mathrm{b}}$ & $\mathrm{An}(\mathrm{Pe})$ & $12 / 35$ & 0.9 & 0.63 \\
\hline & Anthemis sp. ${ }^{\mathrm{b}}$ & & $1 / 4$ & 0.1 & 0.01 \\
\hline & Anthemis tinctoria & $\mathrm{Bi}$ & $3 / 3$ & 0.1 & 0.01 \\
\hline & Artemisia vulgaris ${ }^{\mathrm{b}}$ & $\mathrm{Pe}$ & $36 / 109$ & 2.8 & 5.90 \\
\hline & Centaurea sp. ${ }^{\mathrm{b}}$ & & $2 / 4$ & 0.1 & 0.01 \\
\hline & Chamomilla recutita $^{\mathrm{b}}$ & An & $1 / 1$ & 0.0 & 0.00 \\
\hline & Chamomilla suaveolens & An & $2 / 8$ & 0.2 & 0.02 \\
\hline & Chondrilla juncea $^{\mathrm{b}}$ & $\mathrm{Pe}$ & $16 / 97$ & 1.7 & 2.33 \\
\hline & Cichorium intybus ${ }^{\mathrm{b}}$ & $\mathrm{Pe}$ & $22 / 81$ & 2.1 & 2.68 \\
\hline & Cirsium arvense $^{\mathrm{b}}$ & $\mathrm{Pe}$ & $12 / 23$ & 0.6 & 0.41 \\
\hline & Cirsium sp. ${ }^{\mathrm{b}}$ & & $7 / 8$ & 0.2 & 0.08 \\
\hline & Conyza canadensis & An & $4 / 15$ & 0.4 & 0.09 \\
\hline & Crepis sp. & & $1 / 7$ & 0.2 & 0.01 \\
\hline & Echinops ritro $^{\mathrm{b}}$ & $\mathrm{Pe}$ & $9 / 19$ & 0.5 & 0.26 \\
\hline & Lactuca serriola ${ }^{\mathrm{b}}$ & $\mathrm{Bi}(\mathrm{An})$ & $5 / 20$ & 0.5 & 0.15 \\
\hline & Picris echioides & An & $36 / 46$ & 1.2 & 2.49 \\
\hline & Picris sp. & & $19 / 28$ & 0.7 & 0.80 \\
\hline & Senecio vulgaris ${ }^{\mathrm{b}}$ & An & $1 / 65$ & 1.7 & 0.10 \\
\hline & Silybum marianum ${ }^{\mathrm{b}}$ & $\mathrm{Bi}$ & $1 / 1$ & 0.0 & 0.00 \\
\hline & Sonchus asper ${ }^{\mathrm{b}}$ & $\mathrm{An}(\mathrm{Bi})$ & $2 / 6$ & 0.2 & 0.02 \\
\hline & Sonchus oleraceus ${ }^{\mathrm{b}}$ & An $(\mathrm{Bi})$ & $15 / 15$ & 0.4 & 0.34 \\
\hline & Sonchus sp. & & $27 / 68$ & 1.8 & 2.76 \\
\hline & Xanthium spinosum ${ }^{\mathrm{b}}$ & An & $11 / 36$ & 0.9 & 0.60 \\
\hline & Xanthium strumarium ${ }^{\mathrm{b}}$ & An & $24 / 51$ & 1.3 & 1.84 \\
\hline \multirow[t]{2}{*}{ Cruciferae } & Capsella bursa-pastoris ${ }^{\mathrm{b}}$ & $\mathrm{Bi}$ & $16 / 142$ & 3.6 & 3.42 \\
\hline & Sinapis arvensis & An & $1 / 6$ & 0.2 & 0.01 \\
\hline \multirow[t]{2}{*}{ Dispacaceae } & Scabiosa sp. & & $13 / 32$ & 0.6 & 0.63 \\
\hline & Scabiosa tenuiis ${ }^{\mathrm{b}}$ & An (Bi) & $3 / 37$ & 0.9 & 0.17 \\
\hline \multirow[t]{2}{*}{ Geraniaceae } & Erodium ciconium & $\mathrm{An}(\mathrm{Bi})$ & $1 / 13$ & 0.3 & 0.02 \\
\hline & Erodium sp. & & $1 / 5$ & 0.1 & 0.01 \\
\hline \multirow[t]{5}{*}{ Labiatae } & Ballota nigra & $\mathrm{Pe}$ & $34 / 115$ & 2.9 & 5.88 \\
\hline & Lamium purpureum $^{\mathrm{b}}$ & An & $2 / 18$ & 0.5 & 0.05 \\
\hline & Lycopus europaeus & $\mathrm{Pe}$ & $1 / 8$ & 0.0 & 0.01 \\
\hline & Mentha microphylla & $\mathrm{Pe}$ & $31 / 44$ & 1.2 & 2.05 \\
\hline & Mentha suaveolens & $\mathrm{Pe}$ & $1 / 5$ & 0.1 & 0.01 \\
\hline Malvaceae & Malva neglecta & An & $2 / 24$ & 0.6 & 0.07 \\
\hline \multirow[t]{2}{*}{ Papaveraceae } & Fumaria officinalis ${ }^{\mathrm{b}}$ & An & $1 / 40$ & 1.0 & 0.06 \\
\hline & Papaver rhoeas ${ }^{\mathrm{b}}$ & An & $2 / 105$ & 2.7 & 0.32 \\
\hline Plantaginaceae & Plantago lanceolata & $\mathrm{Pe}$ & $24 / 140$ & 3.6 & 5.05 \\
\hline & Plantago major ${ }^{\mathrm{b}}$ & $\mathrm{Pe}$ & $40 / 151$ & 3.9 & 9.08 \\
\hline Poaceae & Avena fatua & An & $1 / 8$ & 0.2 & 0.01 \\
\hline & Lolium perrene & & $1 / 13$ & 0.3 & 0.02 \\
\hline Polygonaceae & Polygonum aviculare & An & $1 / 8$ & 0.2 & 0.01 \\
\hline & Rumex sp. & An & $13 / 40$ & 1.0 & 0.78 \\
\hline Ranunculaceae & Clematis flammula ${ }^{\mathrm{b}}$ & $\mathrm{Pe}$ & $1 / 4$ & 0.1 & 0.01 \\
\hline & Clematis vitalba & $\mathrm{Pe}$ & $4 / 19$ & 0.5 & 0.11 \\
\hline Rosaceae & Potentilla reptans & $\mathrm{Pe}$ & $14 / 69$ & 1.8 & 1.45 \\
\hline & Sanguisorba minor ${ }^{\mathrm{b}}$ & $\mathrm{Pe}$ & $30 / 55$ & 1.4 & 2.48 \\
\hline Rubiaceae & Galium lucidum & $\mathrm{Pe}$ & $4 / 58$ & 1.5 & 0.35 \\
\hline Scrophulariaceae & Veronica chamaedrys ${ }^{\mathrm{b}}$ & $\mathrm{Pe}$ & $3 / 4$ & 0.1 & 0.02 \\
\hline & Veronica officinalis ${ }^{\mathrm{b}}$ & $\mathrm{Pe}$ & $1 / 1$ & 0.0 & 0.00 \\
\hline & Veronica persica $^{\mathrm{b}}$ & An & $1 / 23$ & 0.6 & 0.03 \\
\hline Solanaceae & Datura stramonium $^{\mathrm{b}}$ & An & $45 / 106$ & 2.7 & 7.17 \\
\hline & Solanum nigrum ${ }^{\mathrm{b}}$ & An & $101 / 229$ & 5.9 & 34.77 \\
\hline Verbenaceae & Verbena officinalis & $\mathrm{Pe}$ & $2 / 5$ & 0.2 & 0.02 \\
\hline Zygophylaceae & Tribulus terrestris & An & $3 / 21$ & 0.5 & 0.09 \\
\hline
\end{tabular}

a Newly identified host species are given in bold.

b Infection was confirmed by mechanical inoculation to petunia and $N$. rustica. 
vulgaris; five plants each of Chenopodium album and Clematis flamula; 10 plants of Aristolochia clematitis and Portulaca oleraceae; and 14 of Heliotropium europaeum plants sampled for the presence of thrips in the tobacco fields.

Infectivity of thrips infesting weed plants. Thrips infesting weeds in the tobacco field showed various levels of numbers of thrips were found on some species such as Amaranthus and Descurainia. High transmission rates were obtained by thrips from infected plants of the species Artemisia, Heliotropium, Chondrila, Datura, and Sonchus. Infectious thrips were collected from healthy plants of Portulaca, Chenopodium, Xanthium, and Amaranthus and from plants of the nonsusceptible species Echinochloa, Descurainia, Rubus, Centaurea, and Kichxia (Table 3).

None of the thrips, collected on three Sonchus oleraceus, 10 Conyza cannadense, 10 Senecio vulgaris, 15 Xanthium spinotransmission efficiency (Table 3). High

sum, and 50 Cirsium arvense healthy plants in the greenhouse, appeared to be viruliferous, even those collected on the seven infected out of 20 Datura stramonium plants. In the same greenhouse, chrysanthemum flowers were infested with high numbers of $F$. occidentalis individuals. They differed considerably in their infection rate when sampled from individual flowers of a red $(24.5 \%)$ versus a white cultivar (42\%).

\section{DISCUSSION}

Weeds may serve as reservoirs for many plant viruses and their vectors $(15,34)$. Their role as reservoirs for TSWV and hosts of thrips to infect nearby susceptible crops has been the subject of several studies $(11,12,19,21,33)$. The present study is an attempt to record the presence of TSWV in the native flora of Greece by analyzing the occurrence of its hosts in and around greenhouse crops and tobacco fields.

A number of weed species either occurring in the greenhouse or tobacco fields

Table 3. Infectivity of thrips collected from individual weeds in tobacco crops $^{\mathrm{a}}$

\begin{tabular}{|c|c|c|}
\hline Weed species & Number of plants sampled & Thrips infected/collected \\
\hline$\overline{\text { Amaranthus albus }}{ }^{\mathrm{b}}$ & 2 & $4 / 39$ \\
\hline Amaranthus albus ${ }^{\mathrm{b}, \mathrm{d}}$ & 2 & $1 / 40$ \\
\hline Amaranthu bletoides ${ }^{\mathrm{d}}$ & 1 & $0 / 11$ \\
\hline Amaranthu retroflexus ${ }^{\mathrm{d}}$ & 9 & $13 / 61$ \\
\hline Anthemis tinctoria ${ }^{\mathrm{b}}$ & 1 & $0 / 1$ \\
\hline Artemisia vulgaris ${ }^{\mathrm{b}}$ & 2 & $2 / 3$ \\
\hline Chenopodium album & 8 & $10 / 36$ \\
\hline Convolvulus arvensis ${ }^{\mathrm{b}, \mathrm{d}}$ & 2 & $1 / 15$ \\
\hline Convolvulus arvensis ${ }^{\mathrm{c}, \mathrm{d}}$ & 2 & $0 / 8$ \\
\hline Conyza canadense & 4 & $1 / 9$ \\
\hline Cynodon dactylon & 2 & $0 / 4$ \\
\hline Centaurea diffusa ${ }^{\mathrm{d}}$ & 2 & $2 / 11$ \\
\hline Cichorium intybus $^{\mathrm{b}}$ & 1 & $0 / 1$ \\
\hline Cichorium intybus ${ }^{\mathrm{c}}$ & 11 & $1 / 68$ \\
\hline Chondrila juncea $a^{\mathrm{b}, \mathrm{d}}$ & 3 & $7 / 24$ \\
\hline Chondrila juncea ${ }^{\mathrm{c}, \mathrm{d}}$ & 3 & $2 / 23$ \\
\hline Consolida regalis $\mathrm{d}$ & 1 & $0 / 1$ \\
\hline Descurainia sophia & 6 & $24 / 105$ \\
\hline Datura stramonium ${ }^{\mathrm{b}}$ & 12 & $13 / 48$ \\
\hline Erignium campestre $\mathrm{d}^{\mathrm{d}}$ & 1 & $0 / 36$ \\
\hline Echinochloa crus-galli & 1 & $3 / 13$ \\
\hline Echinop ritro $^{\mathrm{b}, \mathrm{d}}$ & 1 & $0 / 56$ \\
\hline Echinops ritro $^{\mathrm{c}}$ & 2 & $5 / 76$ \\
\hline Heliotropium europaeum ${ }^{\mathrm{b}, \mathrm{d}}$ & 1 & $1 / 2$ \\
\hline Heliotropium europaeum $\mathrm{c}, \mathrm{d}$ & 6 & $1 / 36$ \\
\hline Kichxia elatine & 2 & $4 / 35$ \\
\hline Lactuca serriola & 1 & $0 / 2$ \\
\hline Mentha swaveolens & 1 & $0 / 4$ \\
\hline Portulaca oleraceae & 3 & $2 / 3$ \\
\hline Picris sp. & 1 & $0 / 1$ \\
\hline Rubus sp. & 2 & $2 / 9$ \\
\hline Sonchus arvensis & 5 & $2 / 94$ \\
\hline Solanum nigrum ${ }^{\mathrm{b}, \mathrm{d}}$ & 1 & $0 / 2$ \\
\hline Solanum nigrum ${ }^{\mathrm{c}, \mathrm{d}}$ & 7 & $2 / 21$ \\
\hline Sonchus oleraceus ${ }^{\mathrm{b}}$ & 1 & $1 / 5$ \\
\hline Sorghum halepense & 2 & $0 / 22$ \\
\hline Tribulus terrestris ${ }^{\mathrm{d}}$ & 4 & $2 / 22$ \\
\hline Xanthium spinosum & 5 & $2 / 27$ \\
\hline Xanthium strumarium ${ }^{\mathrm{b}, \mathrm{d}}$ & 1 & $0 / 3$ \\
\hline Xanthium strumarium ${ }^{\mathrm{c}, \mathrm{d}}$ & 2 & $1 / 4$ \\
\hline
\end{tabular}

a Thrips were tested in two inoculation access periods of $48 \mathrm{~h}$ on a leaf disk of the tobacco variety Basmas BZ/7.

${ }^{\mathrm{b}}$ Plants positive in enzyme-linked immunosorbent assay (ELISA).

${ }^{c}$ Plants negative in ELISA.

${ }^{\mathrm{d}}$ Indicates the presence of thrips larvae on the plants sampled. were recorded as new hosts of TSWV (Tables 1 and 2), by which the number of susceptible species to TSWV increased by $4 \%$ (23). Most of the newly identified hosts occurred in tobacco fields, in which T. tabaci was the only encountered vector species. This species is the main vector of TSWV on tobacco in eastern Europe $(7,17,41)$, while Frankliniella fusca is considered to be the main vector of this virus on tobacco in the United States (16,20,31). The T. tabaci populations occurring in the United States consist only or mainly of females $(16,31)$, whereas the populations found on tobacco in eastern Europe consists of males and females $(7,41)$. As previously shown, thelokotous populations do not transmit TSWV (37), while arrhenotokous populations from tobacco are more efficient transmitters than those isolated from leeks $(7,10)$. The occurrence of $T$. tabaci on infected weed plants in the tobacco crop strongly suggests that the virus is spread by this species from the native flora to tobacco and vice versa. Nevertheless, the efficiency by which they transmit the virus in both directions is not known.

TSWV-infected plants of many weeds either remained symptomless or outgrew initial symptoms as has previously been observed for several species $(4,19)$ and for other viruses as well $(18,24)$. Except for species-specific properties, the absence of symptoms may be the result of natural selection $(15,25)$ or other factors, such as temperature (28).

Failures to demonstrate the presence of TSWV in infected weeds by inoculation tests as recorded in this study have also been reported elsewhere $(11,33)$ and were attributed to various factors (11). The susceptibility of test plants and their physiological age may also affect the reliability of TSWV bioassays demonstrating the need for proper and sensitive assays to index plants for the presence of TSWV.

Some of the 122 species, on which TSWV could not be detected, may have escaped infection, as they are known to be susceptible to TSWV, or belong to families with species susceptible to TSWV (23). The absence of any infection can be ascribed either to natural immunity to TSWV, or to a repulsion by the prevailing thrips species.

The importance of a plant species as a possible virus source depends both on the percentage of infected plants and their relative occurrence among the local flora composition in the vicinity of susceptible crops. This study showed that those species with a high percentage of infected plants were usually represented by a low number of plants in our samples, whereas low percentages were often in species with high numbers of sample plants. This can lead to over- or underestimation of the role of some species as virus source as made in some species $(4,11,19)$. To estimate the 
significance of the infected plants of a species in the spread of TSWV more reliably, a relative infection potential index was introduced which provides a tool to evaluate the importance of various species as virus sources in the local flora. Nine species had indices higher than one in the greenhouses for a total index sum of $94 \%$. The indices are lower in the tobacco crops reflecting a higher diversity of weeds. Seventeen species had an infection potential index greater than one in the tobacco crop with a total index of 93 (Table 2).

Some plant species were found in both crop systems, but were infected in only one of them. This difference can probably be attributed to the vectors infesting these species in different systems. T. tabaci was mainly found on tobacco and weeds in tobacco fields, whereas $F$. occidentalis occurred predominantly in greenhouses. Infected plants of Solanum elaeagnifolium, a known host of $F$. occidentalis but not of T. tabaci (14), occurred only in greenhouses. T. tabaci was occasionally found on weeds in greenhouses. We did not, however, analyze their ability to transmit TSWV. Their transmission efficiency may differ with the host where T. tabaci populations originate $(7,10,37)$. More comparative studies are needed to clarify the role of T. tabaci found on tobacco or other plants in the spread of TSWV in greenhouses.

Since the virus has to be acquired by larvae $(31,36)$, virus dissemination occurs from plants that are hosts of the virus and thrips and support thrips oviposition and reproduction. Capsela bursa-pastoris, Rumex crispus $(11,33)$, Chenopodium album, Sonchus oleraceus, Portulaca oleraceae, Cirsium arvene, Trifolium sp., Lamium amplexicaule, Solanum nigrum (6), Datura stramonium $(2,6)$, and Solanum elaeagnifolium (14), found in the greenhouses, are known hosts of $F$. occidentalis which may disseminate TSWV from these plant species in the greenhouse to susceptible crops. This species also feeds on species of the genera Centaurea (6) and Malva $(2,39)$ which appeared to be susceptible to TSWV as shown in this study. Overwintering hosts such as Amaranthus retroflexus, Conyza canadensis, Portulaca oleraceae (29), Chenopodium album, Sonchus oleraceus, and Lamium amplexicaule (13), as well as other known hosts of T. tabaci such as Verbena (29), Senecio, and Ipomea (14), were identified as hosts of TSWV.

Other properties of a species such as their attractiveness for thrips and suitability for their reproduction (1), the period in which they bloom or become infected, and their life span are also factors affecting virus spread. In blooming chrysanthemum plants, host preference for the pollen feeder $F$. occidentalis (40) over its neighboring weeds was observed, whereas weeds were preferred when the chrysanthemum did not bloom. Infected plants of the species
Chondrila juncea, Datura stramonium, Heliotropium europaeum, and Sonchus oleraceus were infested by high numbers of T. tabaci. Preference for a host may be enhanced by the virus infection $(3,5)$ possibly due to induced biochemical changes (32). Nevertheless, highly preferred hosts may limit migration, hence, spread of TSWV (36).

In addition to thrips (6), the virus can also overwinter in biennials and perennial species from which spread may occur in the next season. These weeds can be a critical factor in TSWV epidemiology especially in tobacco crops as they are the only plants that survive between cropping seasons. Migration of the vectors from weed sources into cultivated fields is stimulated by such factors as maturity or wilting of weeds (30) or human activity (34).

The results of this study underline the need to reduce the number of weeds in greenhouse as well as in tobacco crop systems in order to manage the spread of TSWV. This spread may be further controlled by eliminating weeds that are hosts of the vectors (42). Nevertheless, more extensive studies are required on the relationships between weeds as thrips hosts and virus source to elucidate their impact on the survival and spread of TSWV and to prevent high crop losses.

\section{LITERATURE CITED}

1. Allen, W. R., and Broadbent, A. B. 1986. Transmission of tomato spotted wilt virus in Ontario greenhouses by Frankliniella occidentalis. Can. J. Plant. Pathol. 8:33-38.

2. Bautista, R. C., and Mau, R. F. L. 1994. Preferences and development of western flower thrips (Thysanoptera: Thripidae) on plant hosts of tomato spotted wilt tospovirus in Hawaii. Environ. Entomol. 23:1501-1507.

3. Bautista, R. C., Mau, R. F. L., Cho, J. J., and Custer, D. M. 1995. Potential of tomato spotted wilt tospovirus plant hosts in Hawaii as virus reservoirs for transmission by Frankliniella occidentalis (Thysanoptera: Thripidae). Phytopathology 85:953-958.

4. Bitterlich, I., and MacDonald, L. S. 1993. The prevalence of tomato spotted wilt virus in weeds and crops in southern British Columbia. Can. Plant Dis. Surv. 73:137-142.

5. Carter, W. 1939. Population of Thrips tabaci, with special reference to virus transmission. J. Anim. Ecol. 8:261-276.

6. Chamberlin, J. R., Todd, J. W., Beshear, R. J., Culbreath, A. K., and Demski, J. W. 1992. Overwintering hosts and wingform of thrips, Frankliniella spp., in Georgia (Thysanoptera: Thripidae): Implications for management of spotted wilt disease. Environ. Entomol. 21:121-128.

7. Chatzivassiliou, E. K., Katis, N. I., and Peters, D. 1998. Transmission of tomato spotted wilt virus (TSWV) by Thrips tabaci grown on tobacco or non-tobacco crops. Pages 59-62 in: Proc. Int. Symp. Tospovirus Thrips Floral Vegetable Crops, 4th. Wageningen, the Netherlands.

8. Chatzivassiliou, E. K., Livieratos, I., Avgelis, A., Katis, N. I., and Lykouressis, D. 1996. Occurrence of tomato spotted wilt virus in vegetable and ornamental crops in Greece. Acta Hortic. 431:49-54.

9. Chatzivassiliou E. K., Livieratos, I., Jenser,
G., and Katis, N. I. 2000. Ornamental plants and thrips populations associated with tomato spotted wilt virus in Greece. Phytoparasitica 28:257-264.

10. Chatzivassiliou, E. K., Nagata, T., Katis, N. I., and Peters, D. 1999. The transmission of tomato spotted wilt tospovirus (TSWV) by Thrips tabaci Lind. (Thysanoptera: Thripidae) populations originating from leek. Plant Pathol. 48:700-706

11. Cho, J. J., Mau, R. F. I., Gonsalves, D., and Mitchell, W. C. 1986. Reservoir weed hosts of tomato spotted wilt virus. Plant Dis. 70:10141017.

12. Cho, J. J., Mau, R. F. L., Mitchell, W. C., Gonsalves, D., and Yudin, L. S. 1987. Host list of plants susceptible to tomato spotted wilt virus (TSWV). Res. Ext. Ser. 078, HITAHR. University of Hawaii, Honolulu.

13. Cho, K., Eckel, C. S., Walgenbach, J. F., and Kennedy, G. G. 1995. Overwintering of thrips (Thysanoptera: Thripidae) in North Carolina. Environ. Entomol. 24:58-67.

14. Doederlein, T. A., and Sites, R. W. 1993. Host plant preferences of Frankliniella occidentalis and Thrips tabaci (Thysanoptera: Thripidae) for onions and associated weeds on the Southern high plains. J. Econ. Entomol. 86:1706-1713.

15. Duffus J. E. 1971. Role of weeds in the incidence of virus diseases. Annu. Rev. Phytopathol. 9:319-340.

16. Eckel, S. C., Kijong, C., Walgenbach, J. F. Kennedy, G., and Moyer, W. 1996. Variation in thrips species composition in field crops and implications for the tomato spotted wilt epidemiology in North Carolina. Entomol. Exp. Appl. 78:19-29.

17. Jenser, G., and Gáborjányi, R. 1998. Ecological aspects of tomato spotted wilt epidemy in Hungary. Pages 81-82 in: Proc. Int. Symp. Tospovirus Thrips Floral Vegetable Crops, 4th. Wageningen, the Netherlands.

18. Khan, M. A., Hibino, H., Aguiero, V. M., Daquioag, R. D., and Opina, O. S. 1991. Rice and weed hosts of rice tungro-associated viruses and leafhopper vectors. Plant Dis. 75:926-930.

19. Latham, L. J., and Jones, R. A. C. 1997. Occurrence of tomato spotted wilt tospovirus in native flora, weeds and horticultural crops. Aust. J. Agric. Res. 48:359-369.

20. McPherson, R. M., Pappu, H. R., and Jones, D. C. 1999. Occurrence of five thrips species in flue-cured tobacco and impact on spotted wilt disease incidence in Georgia. Plant Dis. 83:765-767.

21. Van Os, B., Stancanelli, G., Mela, L., and Lisa, V. 1993. Role of wild plants and weeds in the epidemiology of tospoviruses in Liguria. Inf. Fitopatol. 42:40-44.

22. Palmer, J. M., Mound, L. A., and Du Heaume, G., J. 1992. IIE guides to insects of importance to man: 2. Thysanoptera. C.A.B. International, Wallingford, UK.

23. Peters, D. 1998. An updated list of plant species susceptible to tospoviruses. Pages 107-110 in: Proc. Int. Symp. Tospovirus Thrips floral vegetable crops, 4th. Wageningen, the Netherlands.

24. Powell, C. A., Forer, L. B., Stouffer, R. F., Cummins, J. N., Gonsalves, D., Rosenberger D. A., Hoffman, J., and Lister, R. M. 1984. Orchard weeds as hosts of tomato ringspot and tobacco ringspot viruses. Plant Dis. 68:242-244.

25. Power, A. G. 1992. Patterns of virulence and benevolence in insect-borne pathogens of plants. Critic. Rev. Plant Sci. 11:351-372.

26. Reddy, D. V. R., and Wightman, J. A. 1988. Tomato spotted wilt virus: Thrips transmission and control. Adv. Dis. Vector Res. 5:203220. 
27. Roditakis, N. 1991. First record of Frankliniella occidentalis in Greece. Entomol. Hellenic. 9:77-79.

28. Roggero, P., and Pennazio, S. 1997. Thermal inactivation of tomato spotted wilt tospovirus in vivo. Physiol. Mol. Plant Pathol. 51:35-40.

29. Sakimura, K. 1932. Life history of Thrips tabaci L. on Emilia sagittata and its host plant range in Hawaii. J. Econ. Entomol. 25:884-891.

30. Sakimura, K. 1961. Field observations on the thrips vector species of the tomato spotted wilt virus in the San Pablo area, California. Plant Dis. Rep. 45:772-776.

31. Sakimura, K. 1962. The present status of thrips-borne viruses. Pages 33-40 in: Biological Transmission of Disease Agents. K. Maramorosch, ed. Academic Press, New York.

32. Selman, I. W., Brierley, G. F., Pegg, G. F., and Hill, T. A. 1961. Changes in the free amino acids and amides in tomato plants inoculated with tomato spotted wilt virus. Ann. Appl.
Biol. 49:601-615.

33. Stobbs, L. W., Broadbent, A. B., Allen, W. R., and Stirling, A. L. 1992. Transmission of tomato spotted wilt virus by the western flower thrips to weeds and native plants found in Southern Ontario. Plant Dis. 76:13-29.

34. Thresh, J. M. 1981. The role of weeds and wild plants in the epidemiology of plant virus diseases. Pages 53-70 in: Pests Pathogens and Vegetation. J. M. Thresh, ed. Pitman Publications, London.

35. Tsakiridis J. P., and Gooding, G. V., Jr. 1972. Tomato spotted wilt virus in Greece. Phytopathol. Mediter. 11:42-47.

36. Ullman, D. E., Cho, J. J., Mau, R. F. L., Hunter, W. B., Westcott, D. M., and Custer, D. M. 1992. Thrips-tomato spotted wilt virus interaction: Morphological, behavioral and cellular components influencing thrips transmission. Adv. Dis. Vector Res. 9:195-240.

37. Wijkamp, I., Alarza, N., Goldbach, R., and Peters, D. 1995. Distinct levels of specificity in thrips transmission of tospoviruses. Phyto- pathology 85:1069-1074.

38. Wijkamp, I., and Peters, D. 1993. Determination of the median latent period of two tospoviruses in Frankliniella occidentalis, using a novel leaf disk assay. Phytopathology 82:986-991.

39. Yudin, L. S., Cho, J. J., and Mitchell, W. C. 1986. Host range of western flower thrips, Frankliniella occidentalis (Thysanoptera: Thripidae), with special reference to Leucaena glauca. Environ. Entomol. 15:1292-1295.

40. Yudin, L. S., Tabashnik, B. E., Cho, J. J., and Mitchell, W. C. 1988. Colonization of weeds and lettuce by thrips (Thysanoptera: Thripidae). Environ. Entomol. 17:522-526.

41. Zawirska, I. 1978. Studies on Thrips tabaci Lindeman (Thysanoptera: Thripidae). Pr Nauk. Inst. Ochr. Rosl. 2:115-138.

42. Zitter, T. A., and Simons, J. N. 1980. Management of viruses by alteration of vector efficiency and by cultural practices. Annu. Rev. Phytopathol. 18:289-310. 